\title{
Clinical validation of time reduction strategy in continuous step-and-shoot mode during SPECT acquisition
}

\author{
Valentin Picone ${ }^{1}$, Nikolaos Makris ${ }^{1}$, Fanny Boutevin ${ }^{1}$, Sarah Roy ${ }^{1}$, Margot Playe ${ }^{2}$ and Michael Soussan ${ }^{2,3^{*}}$ (D)
}

\author{
* Correspondence: Michael. \\ soussan@aphp.fr \\ ${ }^{2}$ Department of Nuclear Medicine, \\ Avicenne Hospital, HUPSSD, APHP, \\ Paris, France \\ ${ }^{3}$ Inserm, Institut Curie, Laboratoire \\ d'Imagerie Translationnelle en \\ Oncologie, Orsay, France \\ Full list of author information is \\ available at the end of the article
}

\begin{abstract}
Background: The SwiftScan solution (General Electric Healthcare) combines a new low-energy high-resolution sensitivity collimator and a tomographic step-and-shoot continuous (SSC) mode acquisition. The purpose of this study is to determine whether SSC mode can be used in clinical practice with shorter examination times, while preserving image quality and ensuring accurate semi-quantification. Twenty bone scan and 10 lung scan studies were randomly selected over a period of 2 months. Three sets of image datasets were produced: step-and-shoot (SS) acquisition, simulated 25\% count reduction using the Poisson resampling method (SimSS), and SimSS continuous acquisition (SimSSC), where SimSS was summed with counts acquired during detector head rotation. Visual assessment (5-point Likert scale, 2 readers) and semi-quantitative evaluation (50 focal uptake from 10 bone

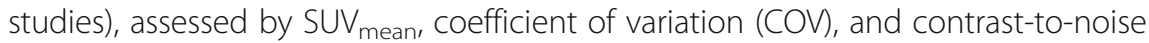
ratio (CNR), were performed using $t$ test and Bland-Altman analysis.
\end{abstract}

Results: Intra-reader agreement was substantial for reader $1(k=0.71)$ and for reader $2(k=0.61)$. Inter-reader agreement was substantial for SS set $(k=0.93)$ and moderate for SimSSC $(k=0.52)$. Bland-Altman analysis showed a good interchangeability of SS and SimSSC SUV values. The mean CNR between SS and SimSSC was not significantly different: $42.9 \pm 43.7$ [23.7-62.1] vs. $43.1 \pm 46$ [22.9-63.3] $(p=0.46)$, respectively. COV values, assessing noise level, did not deviate significantly between SS and SimSSC: $0.20 \pm 0.08$ [0.18-0.23] vs. $0.21 \pm 0.08$, [0.18-0.23] ( $p=0.15)$, respectively, whereas a significant difference was demonstrated between SS and SimSS: $0.20 \pm 0.08$ [0.18-0.23] vs. $0.23 \pm 0.09$ [0.20-0.25] ( $p<0.0001$ ), respectively.

Conclusions: SSC mode acquisition decreases examination time by approximately $25 \%$ in bone and lung SPECT/CT studies compared to SS mode ( 2 min per singlebed SPECT), without compromising image quality and signal quantification. This SPECT sensitivity improvement also offers the prospect of more comfortable exams, with less motion artifacts, especially in painful or dyspneic patients.

Keywords: SPECT/CT, SwiftScan, Lungs, Bone, SUV, Semi-quantification 


\section{Background}

Over the last years, several technological advances have been integrated to singlephoton emission computed tomography (SPECT) [1]. The introduction of computed tomography (CT)-based attenuation correction, scatter correction, and resolution recovery, as well as the introduction of new cadmium zinc telluride (CZT) detectors, has allowed physical phenomena that occur along the pathway of the photon until its detection to be taken into account, rendering semi-quantification in SPECT/CT attainable [2-4]. Besides, different SPECT image acquisition strategies have also been suggested in the nuclear medicine practice. Three modes of SPECT acquisition are available: step-and-shoot (SS), continuous, and step-and-shoot continuous (SSC). In the SS mode, projection data are acquired only when the detector is stationary, whereas in the SSC mode, data are acquired both when the detector is stationary and when the detector moves from one view to the next [5]. During the SSC mode, the system acquires events from three locations: the regular SS position, the half arc immediately preceding the SS position, and the half arc immediately following it. The counts acquired during the $6^{\circ}$ rotation are partitioned as follows: between $0^{\circ}$ and $3^{\circ}$, counts are assigned in position $0^{\circ}$, and between $3^{\circ}$ and $6^{\circ}$, they are integrated in the next position $6^{\circ}$ (Fig. 1).

Although this is a relatively long-standing concept introduced in the mid-90s by Cao et al. [5], who studied the effect in image quality using a SSC versus a conventional SS mode acquisition using computer simulations, only recently was SSC evaluated in a clinical setting. A recent paper of a French team [6] observed on phantoms that SSC mode improves volumetric sensitivity compared to SS mode, without significant impact on image quality. These results suggest that a strategy of time or dose reduction could be applied in a clinical setting.

The SwiftScan solution (General Electric Healthcare) combines a new low-energy high-resolution sensitivity collimator (LEHRS) and a tomographic SSC mode acquisition. The purpose of this study is to determine whether SSC mode can be used in clinical practice with shorter examination times, while preserving image quality and ensuring accurate semi-quantification.

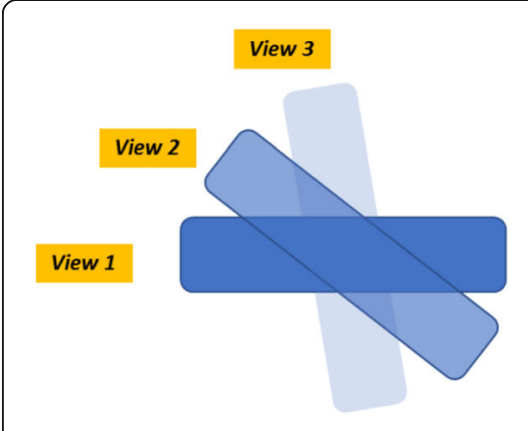

Step and Shoot Mode

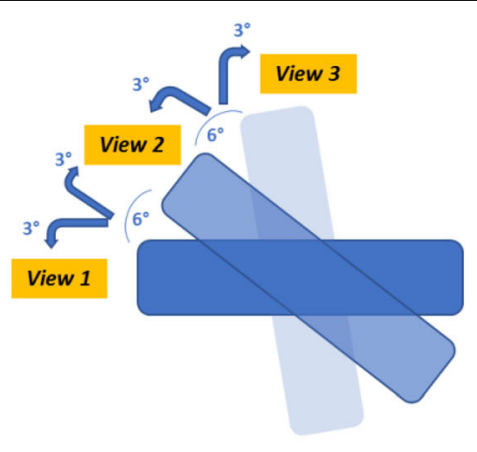

Step and Shot Continuous Mode

Fig. 1 An illustration of step-and-shoot (SS) versus step-and-shoot continuous (SSC) mode acquisition, demonstrating incorporation of counts during the detector head rotation in SSC mode 


\section{Methods}

\section{Patient study}

Twenty patients with 20 bone scan studies and 10 patients with lung scan studies were randomly selected over a period of 2 months between July and November 2018 (Table 1). All studies were performed using a SPECT/CT D870DR gamma camera (GE Healthcare, Waukesha, WI). This gamma camera is coupled to a 16-slice CT scanner and includes latest technological advancements, on iterative reconstruction and metal artifact correction. Bone scans were performed using a double bed position, covering dorsal and lumbar spine, following an administration of $727 \pm 82 \mathrm{MBq}$ of ${ }^{99 \mathrm{~m}} \mathrm{Tc}$-osteocis. For lung scans, the average dose of Technegas administered to the patients was $407 \pm 40 \mathrm{MBq}$ (ventilation) and $193 \pm 9 \mathrm{MBq}$ of ${ }^{99 \mathrm{~m}}$ Tc-labeled albumin macroaggregates (perfusion).

\section{Image acquisition and reconstruction protocols}

The SPECT acquisition protocols were performed with a two-head camera, with 60 projections over $360^{\circ}, 180^{\circ}$ per detector, step of $6^{\circ}, 15$ and $16 \mathrm{~s} /$ projection for bone and lung studies, respectively.

All images were reconstructed on a Xeleris $4 D R$ workstation with the following parameters:

For bone studies: ordered subsets expectation maximization reconstruction (OSEM) algorithm with 6 subsets, 3 iterations, and a Gaussian filter (FWHM $=5.3 \mathrm{~mm}$ ). Each bone image was corrected for attenuation, scatter, and resolution recovery based on collimator-detector pair response.

For lung studies: OSEM algorithm with 2 iterations, 8 subsets, and a Butterworth filter with $f_{\text {cut }}=0.6 \mathrm{cycle} / \mathrm{cm}$ and $p=5$. We intended to only visually assess these lung cases; thus, no quantitative corrections were applied.

Table 1 Characteristics of the patients

\begin{tabular}{|c|c|}
\hline \multicolumn{2}{|l|}{ Patients with bone scans $(n=20)$} \\
\hline Weight (kg, mean \pm SD) & $70,6 \pm 13,7$ \\
\hline Osteocis ${ }^{\circledast}$ administered activity (MBq, mean \pm SD) & $727 \pm 82$ \\
\hline Time to acquisition (minutes, mean \pm SD) & $228 \pm 47$ \\
\hline \multicolumn{2}{|l|}{ Indications } \\
\hline Bone metastatic disease assessment & $7 / 20$ \\
\hline Bone pain/arthralgias & $6 / 20$ \\
\hline Suspicion of prosthesis loosening or infection & $5 / 20$ \\
\hline Suspicion of complex regional pain syndrome & $2 / 20$ \\
\hline \multicolumn{2}{|l|}{ Patients with lung scans $(n=10)$} \\
\hline \multicolumn{2}{|l|}{ Administered activity (MBq, mean $\pm \mathrm{SD}$ ) } \\
\hline Technegas $^{\circledR}$ (ventilation) & $407 \pm 40$ \\
\hline Pulmocis $^{\circledast}$ (perfusion) & $193 \pm 9$ \\
\hline \multicolumn{2}{|l|}{ Indications } \\
\hline Suspected pulmonary embolism & $9 / 10$ \\
\hline Pre-operative evaluation & $1 / 10$ \\
\hline
\end{tabular}


In order to simulate a decrease in time reduction, low count statistic images were reconstructed with a $25 \%$ time reduction in acquisition using the raw data from the SS mode and using a Poisson resampling algorithm [7]. The detector rotation time from projection $N$ to projection $N+1$ is in the order of approximately $2 \mathrm{~s}(\sim 2 \mathrm{~min}$ per single-bed SPECT study). As this study contains bone and lung protocols with time per projection of about $16 \mathrm{~s}$, the selection of $25 \%$ time reduction will even out the addition of counts during head rotation between projections. Lower or higher time per projection acquisitions may slightly increase or decrease, respectively, the projected time gain. Poisson sampling is a process in which each element of the population is subjected to an independent Bernoulli test that determines whether the element becomes part of the sample. Each element of the population may have a different probability of being included in it. This probability when drawing a single sample is identified by the first order inclusion probability of that element. If all first order inclusion probabilities are equal, Poisson sampling becomes equivalent to Bernoulli sampling, which can therefore be considered as a particular case of Poisson sampling. Mathematically, the first-order inclusion probability of the second element of the population is designated by the symbol $\pi_{i}$. The probability of second order inclusion, when a couple consisting of the $i$ th and $j$ th elements of the population sampled, is included in a sample during the formation of a single sample rated $\pi_{\mathrm{ij}}$. The following relationship is valid during Poisson sampling: $\pi_{i j}=\pi_{i} \times \pi_{j}$

\section{Data analysis}

In this study, three set of image datasets were produced as follows (Table 2):

- Set 1. Step-and-shoot (SS) acquisition

- Set 2. Simulated SS (SimSS): simulated 25\% count-reduction using the Poisson resampling method

- Set 3. Simulated SS and continuous (SimSSC) acquisition: set 2 was summed with counts acquired during detector head rotation.

In this study, only set 1 and set 3 are used for both visual assessment and semiquantitative evaluation. Set 2 was produced as an intermediate dataset to create set 3 .

\section{Visual assessment}

The 30 SPECT studies were visually evaluated by two experienced nuclear medicine physician (reader 1: M.S. and reader 2: M.P.). The reviewers compared blindly SS and SimSSC images and attributed a grade using a 5-point ordinal scale (Likert score) [8] to evaluate the image quality based on the following criteria: non-diagnostic image

Table 2 Time parameters for three acquisition modes in bone and lung SPECT/CT protocols

\begin{tabular}{lll}
\hline Acquisition mode/organ & Bone SPECT & Lung SPECT \\
\hline Step-and-shoot (SS) & $16 \mathrm{~s}$ & $15 \mathrm{~s}$ \\
Simulated step-and-shoot (- 25\%) (SimSs) & $12 \mathrm{~s}$ & $11 \mathrm{~s}$ \\
Simulated step-and-shoot (- 25\%) continuous (SimSSC) & $12 \mathrm{~s}+\mathrm{C}$ & $11 \mathrm{~s}+\mathrm{C}$ \\
\hline
\end{tabular}

$C$ : counts acquired during detector rotation between projections 
quality/resolution (grade 0), sub-optimal diagnostic and limited clinical information (grade 1), diagnostic and acceptable image quality/resolution (grade 2), diagnostic and good image quality/resolution (grade 3), and diagnostic and excellent image quality/ resolution (grade 4).

\section{Semi-quantitative evaluation}

For the semi-quantitative evaluation, only bone studies were analyzed. Five lesions were segmented per patient, making a total of 50 volume of interests (VOIs). We chose to use the mean standardized uptake value $\left(\mathrm{SUV}_{\text {mean }}\right)$ instead of $S U V_{\max }$ to avoid the influence of elevated noise in the measurement. The volumes employed as well as their localization, in all three series, were identical. The $\mathrm{SUV}_{\text {mean }}$ was calculated by first selecting the coronal slice with the highest uptake, for each hot spot, in the conventional set of images. Subsequently, a SUV threshold-based segmentation was initiated by placing a seed point on the lesion and the contour was progressively adapted to the lesion boundaries until the threshold criteria were fulfilled. SPECT and CT images were used to employ in a muscle region (e.g., quadriceps) a threshold-based background VOI, encompassing a volume of 10-20 cc. For assessing image noise level, standard deviation of SUV values within each lesion and background VOI was used to calculate the coefficient-of-variation (COV), which was given by the following equation:

$$
\mathrm{COV}=\frac{\mathrm{SD}_{\text {SUVmean }}}{\mathrm{SUV}_{\text {mean }}}
$$

where $\mathrm{SUV}_{\text {mean }}$ and $\mathrm{SD}_{\mathrm{SUV} \text { mean }}$ correspond to the average and standard deviation of within lesion and background VOIs.

The contrast to noise ratio (CNR) was also calculated and was given by the following equation:

$$
\mathrm{CNR}=\frac{N_{\text {lesion }}-N_{\text {background }}}{\sigma_{\text {background }}}
$$

where $N_{\text {lesion }}$ and $N_{\text {background }}$ correspond to the mean activity concentration of the $\mathrm{VOI}_{\text {lesion }}$ and the $\mathrm{VOI}_{\text {background }}$, respectively, and $\sigma_{\text {background }}$ is the standard deviation of the $\mathrm{VOI}_{\text {background. }}$ The $\mathrm{VOI}_{\text {background }}$ was selected in the muscle. All SUV analysis was performed with Q.Volumetrix MI (GE Healthcare, Milwaukee) on a Xeleris 4 DR workstation.

\section{Statistical analysis}

Data are presented as mean \pm standard deviation (SD) [95\% confidence interval]. To assess the consistency of visual assessments, the Cohen's kappa ( $k$ ) [9] for intra-reader and inter-reader agreements was calculated for all scans. Similarity of homogeneity of variance between the quantitative variables of the 3 sets was verified using the Leven's test. The Bland-Altman method [10] was used for comparing $\mathrm{SUV}_{\text {mean }}$ and $\mathrm{SD}_{\text {SUVmean }}$ between SS and SimSSC sets. Mean and SD were reported as well as lower and upper limits of agreement (LOA), calculated as $\pm 1.96 \times \mathrm{SD}$. A Student $t$ test was used to test the null hypothesis at the 0.05 significance level, along with $95 \%$ confidence intervals $(95 \% \mathrm{CI})$; for comparison of the SUV, COV, and CNR between the 3 sets, statistical tests were performed using Excel spreadsheets. 


\section{Results}

\section{Visual comparison between SS and SimSSC images}

Image quality was graded as $3 / 4$ in $90 \%(18 / 20)$ of bone studies and 90\% (9/10) of lung studies for both readers. Intra-reader agreement (between grades 3 and 4) was substantial for reader $1(k=0.71)$ and for reader $2(k=0.61)$. Inter-reader agreement was substantial for SS set $(k=0.93)$ and moderate for SimSSC $(k=0.52)$. Visual examples of image quality of bone and lung studies are shown in Figs. 2 and 3.

\section{Semi-quantitative comparison between SS and SimSSC images}

Semi-quantitative comparison between SS and SimSSC of the 50 VOIs was assessed by means of SUV, COV, and CNR. SUV values were not significantly different between SS and SimSSC: $6.8 \pm 3.3[0.4-13.2]$ vs. $6.5 \pm 3.2[0.2-12.8](p=0.6)$, as well as SD $_{\text {SuVmean }}$ values: $1.4 \pm 1.2[-1-3.8]$ vs. $1.4 \pm 1.1[-0.8-3.6](p=0.9)$. A graphical representation of the data using Bland-Altman plots is shown between SS and SimSSC sets for SUVmean in Fig. 4 and for SD $_{\text {SUVmean }}$ in Fig. 5. The mean difference of SS-SimSSC was $0.3 \pm$ $0.6[-1-1.6]$ for $\mathrm{SUV}_{\text {mean }}$ and $0.04 \pm 0.23$ [- 0.4-0.5] for $\mathrm{SD}_{\mathrm{SUV} \text { mean, }}$ while pooling fifty bone lesions across 10 patients.

In Fig. 6, the use of SimSSC resulted in similar CNR mean values as compared with SS mode: $43.1 \pm 46$ [95\% CI: $22.9-63.3]$ vs. $42.9 \pm 43.7$ [23.7-62.1] $(p=0.46)$ whereas

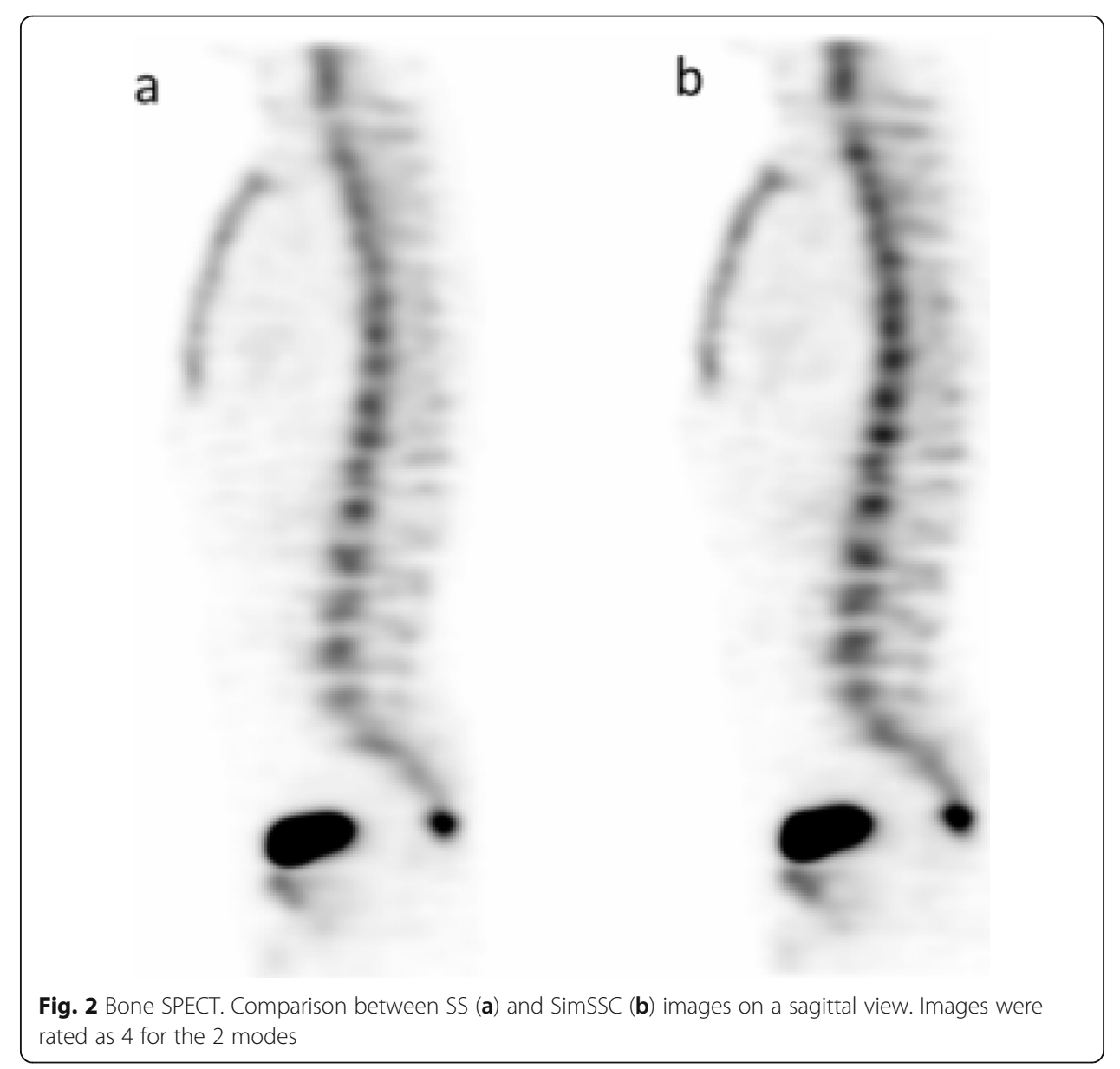


a

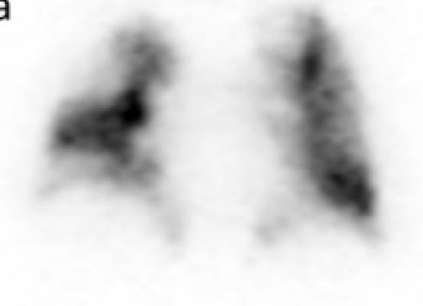

b

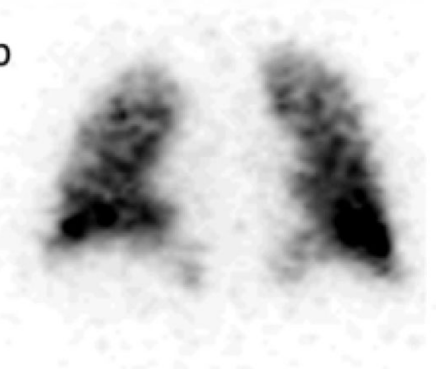

C

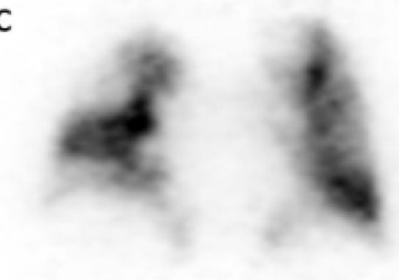

d

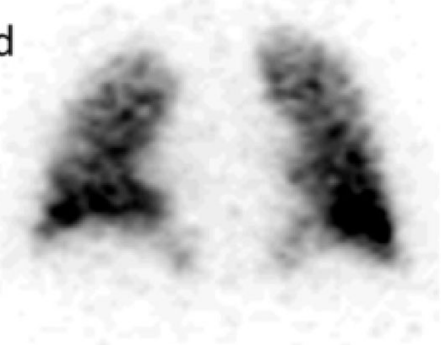

Fig. 3 V/Q lung SPECT. Comparison between SS (a perfusion; b ventilation) and SimSSC (c perfusion; $\mathbf{d}$ ventilation) images on coronal views. Images were rated as 4 for the 2 modes

CNR values with SimSS mode (31.7 \pm 27.1 [19.8-43.6]) differed significantly from SS $(p$ $=0.009)$ and $\operatorname{SimSSC}(p=0.016)$.

Figure 7 shows the average COV obtained using the three acquisition modes. COV mean values did not deviate significantly between SS and SimSSC: $0.20 \pm 0.08$ [95\% CI: 0.180.23 ] vs. $0.21 \pm 0.08$ [0.18-0.23]; ( $p=0.15$ ), whereas a significant difference was demonstrated between SS and SimSS: $0.20 \pm 0.08$ [0.18-0.23] vs. $0.23 \pm 0.09[0.20-0.25](p<0.0001)$.

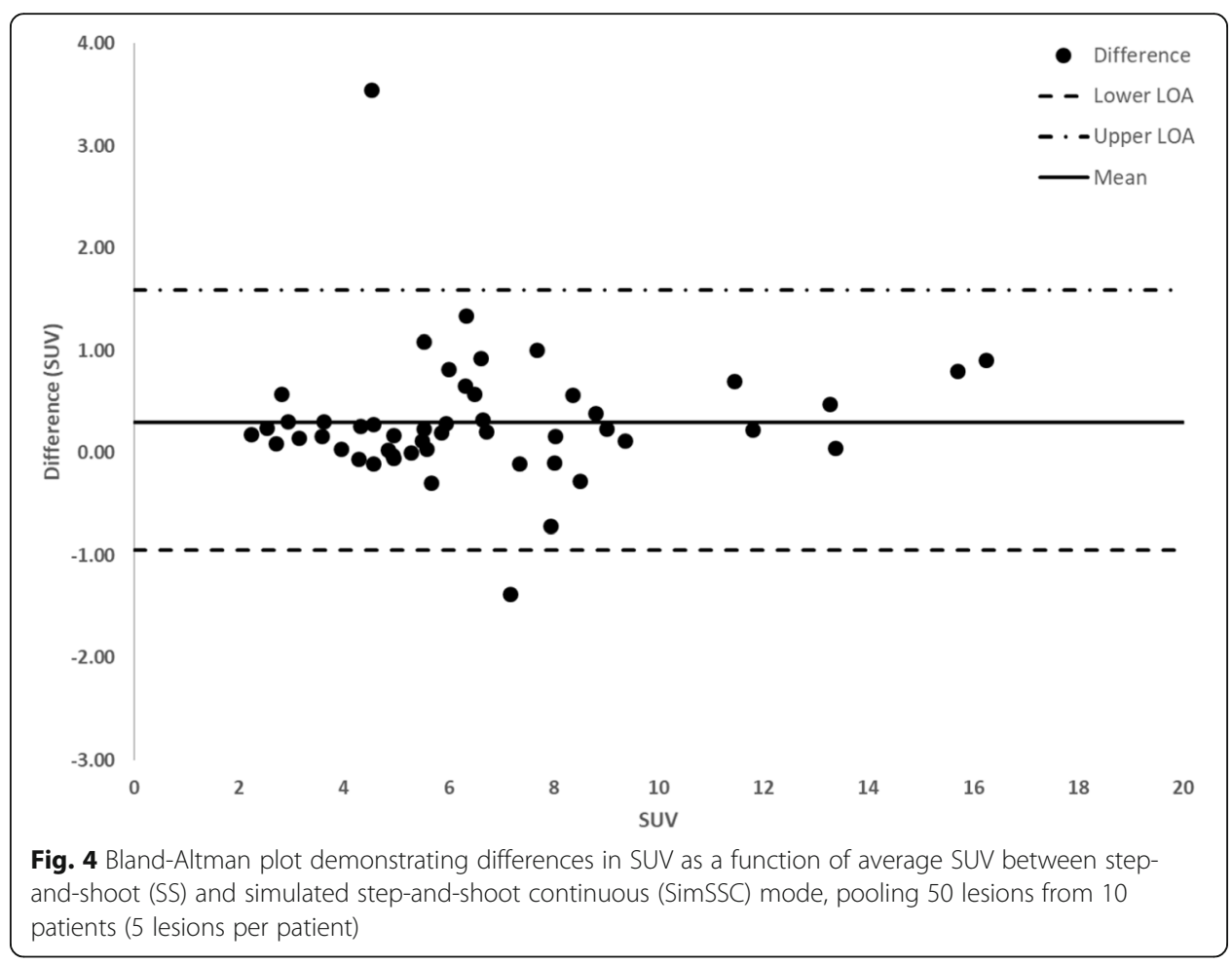




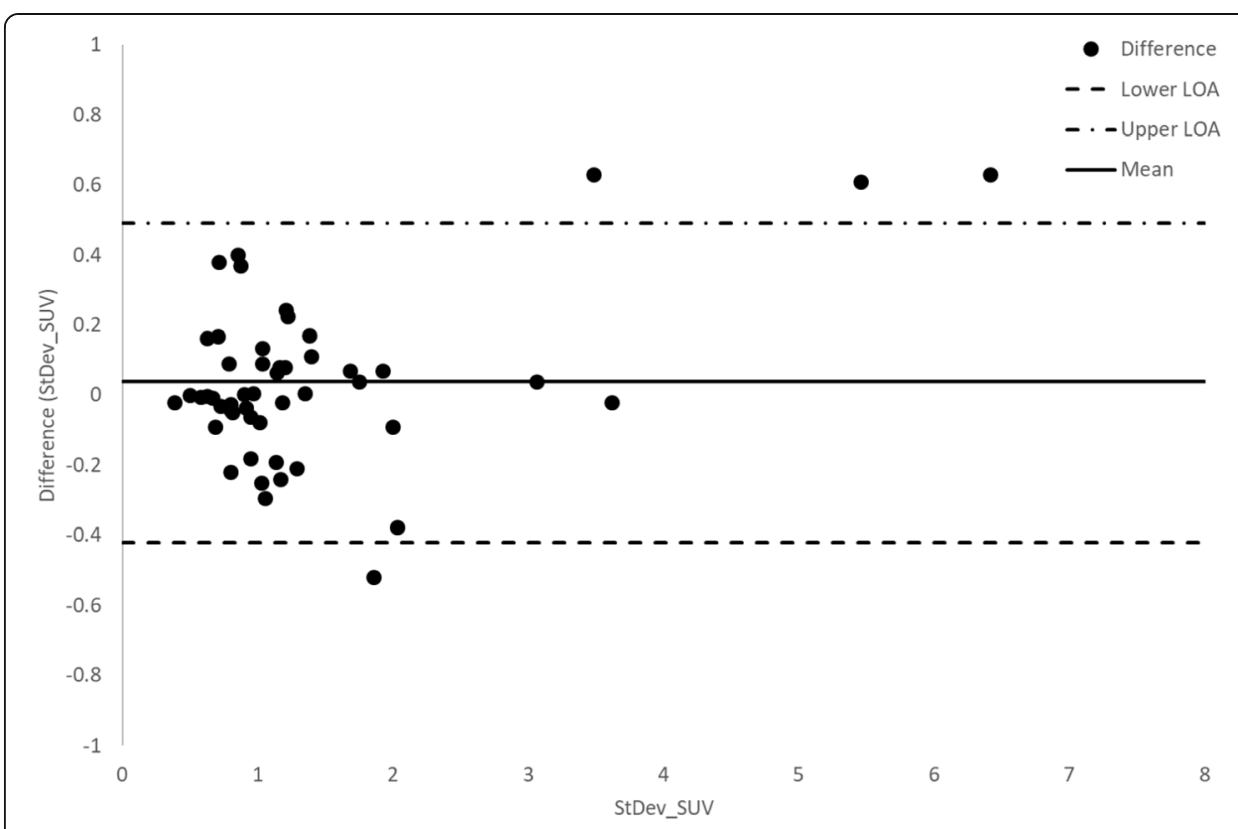

Fig. 5 Bland-Altman plot demonstrating differences in standard deviation of SUV as a function of averaged standard deviation SUV between step-and-shoot (SS) and simulated step-and-shoot continuous mode (SimSSC), pooling 50 lesions from 10 patients

\section{Discussion}

The use of SPECT has been significantly increased these last years. Many strategies have been developed with the aim of reduction of injected activity as well as the time procedure, aiming at improving patient experience and reducing anxiety prior to and during SPECT imaging [11]. Nuclear cardiac imaging benefits the most of these strategies, especially with the introduction of dedicated cardiac cameras [12]. However, in the field of extra cardiac imaging, such as bone or lung studies, there is still a need for

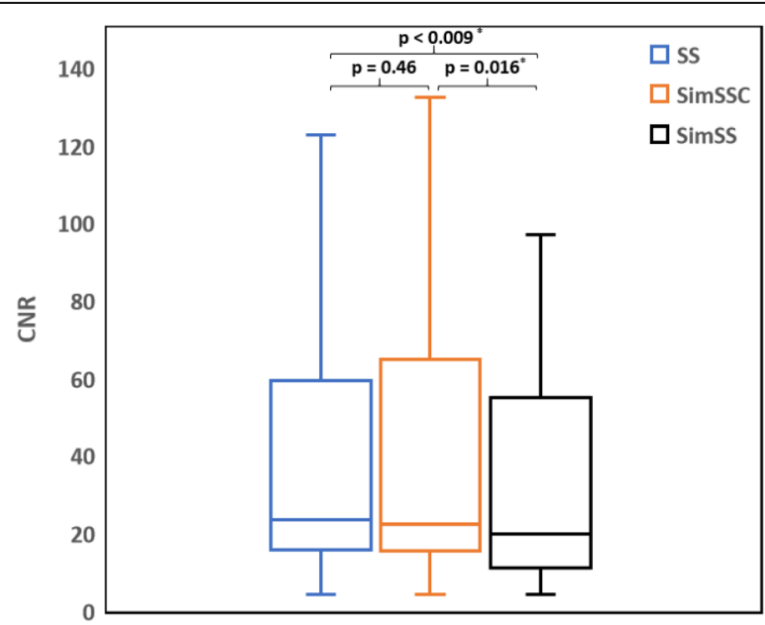

Fig. 6 Box plot on contrast-to-noise ratio for two acquisition modes (SS, step-and-shoot; SimSSC, simulated step-and-shoot continuous; SimSS, simulated step-and-shoot). The median is illustrated by the midline, first and third quartiles by the lower and upper lines of the box, and extremes by whiskers 


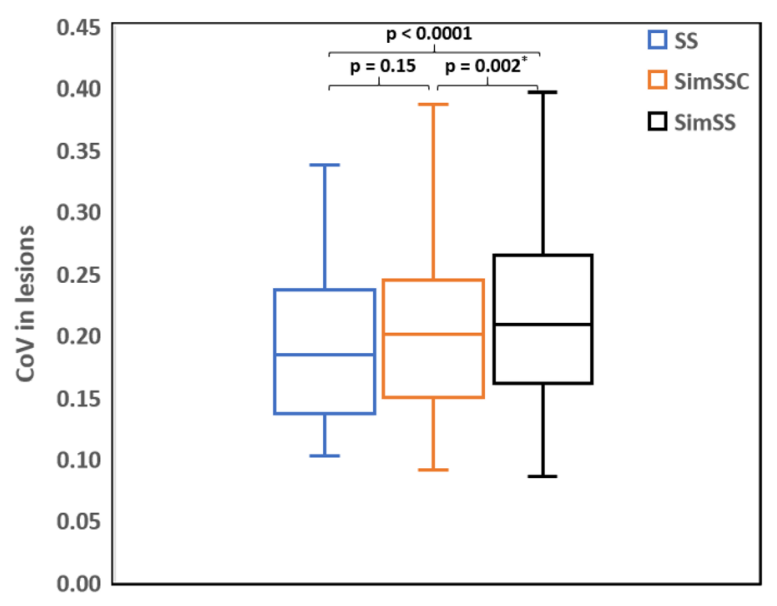

Fig. 7 Box plot showing the coefficient-of-variation of the 50 lesions, for three acquisition modes (SS, stepand-shoot; SimSSC, simulated step-and-shoot continuous; SimSS, simulated step-and-shoot). The median is illustrated by the midline, first and third quartiles by the lower and upper lines of the box, and extremes by whiskers

improvement in SPECT time duration without compromising image quality and quantification.

In this paper, we studied the clinical validity on visual and semi-quantitative assessment of a time reduction strategy during SPECT acquisition using SSC mode acquisition. In general, we demonstrated that incorporation of counts during detector rotation between projections with a $25 \%$ decrease of scan time ( 2 min per single-bed SPECT study) provides an equivalent image quality and accurate quantitative values with respect to standard SS acquisition. In the same way, the SSC mode can also compensate for an equivalent of $25 \%$ dose reduction.

Bland-Altman analysis shows that the vast majority of the SUV values is around the mean line of the differences between SS and SimSSC $(+0.3)$ and almost always between the LOA (except 2 outliers). There is also a small clinically insignificant bias $(+0.3)$ between SS and SimSSC. The difference between LOA is indeed of the order of 2.6 SUV units, which represents about $38 \%$ of the average of SUVs (6.8). The topic of SUV variability in PET imaging has been extensively covered the past decade demonstrating that changes in the acquisition, reconstruction, and post-processing parameters may introduce significant variability in SUV measurements. In clinical practice, if the comparison is made with PET, a threshold of at least $30 \%$ of SUV variation is considered significant and attributable to a biological effect, while also ensuring a meaningful comparability of the two acquisitions. In our study, the difference is of the same order, which points to a non-significant clinical impact of the variability of the measurement between SS and SimSSC. In addition, the use of SUV ratio is also common in SPECT quantification which allows to cancel out plasma clearance and/or cross calibration related differences [13].

These observations show the interchangeability of SUV measurements of SS and SimSSC, as no significant bias was observed, and noise level was not increased in SimSSC.

For both bone and lung studies, visual assessment confirmed the similarity in image quality between SS (bone $16 \mathrm{~s} /$ step; lung perfusion $15 \mathrm{~s} / \mathrm{step}$ ) and SimSSC images (bone 
$12 \mathrm{~s} / \mathrm{step}$; lung perfusion $11 \mathrm{~s} / \mathrm{step}$; lung ventilation $15 \mathrm{~s} / \mathrm{step})$. The noise introduced into the image by the decrease of the counting statistics is well compensated by the activation of the continuous mode. This is corroborated by an excellent COV correlation and no CNR significant differences between SS and SimSSC whereas SimSS demonstrated, as expected, significant different COV values to SS due to the simulated reduction of exam duration. The image quality of bone and lung studies is therefore maintained qualitatively and semi-quantitatively while reducing time examination by $25 \%$ (from $15 \mathrm{~s}$ to $11 \mathrm{~s}$ for lung perfusion and from $16 \mathrm{~s}$ to $12 \mathrm{~s}$ for bone).

Acquiring counts during head rotation is a rather new concept; therefore, only a few publications have been so far appeared in scientific literature. A recent study validated the effects of detector rotation speed and rotation time for continuous repetitive rotation acquisition on image quality and quantification in DaTSCAN SPECT. It was demonstrated that a combination of rotation speed and rotation times affect the image quality and quantification of DaTSCAN SPECT [14]. Overall, the authors suggested the use of added projection data processes and proper rotation speed when continuous repetitive rotation acquisition is applied. In another study by Bailly et al. [13], it was demonstrated that Swiftscan step and shoot continuous acquisition may enable a $25 \%$ time reduction of DaTSCAN acquisitions without changing visual and/or semi-quantitative analysis while reporting on striatal binding SUV ratios. These findings are in agreement with those reported in our study, thus confirming the utility of incorporating additional projections in a SPECT acquisition or demonstrating the possibility for reduced acquisition times without affecting image quality.

Semi quantitative analysis is ready to become a standard in SPECT studies $[4,15]$. The integration of corrections in SPECT imaging such as for photon attenuation and scatter, the use of semi-automatic delineation of volumes of interest and normalization factors (body weight and injected dose) renders semi-quantitative comparison of activity concentrations possible, allowing to reduce SUV variability in follow-up studies. As there is a growing need in SPECT imaging to obtain activity concentrations, this study suggests that SUV quantification and image properties are not altered with the SSC acquisition mode, and therefore, bone and lung SPECT studies can even be performed with shorter duration.

Beyond the gain in scan time or dose reduction, the clinical impact of using SSC instead of conventional SS mode is worth to be evaluated, especially in terms of quantification accuracy, lesion detectability, and characterization.

\section{Conclusions}

Step-and-shoot continuous mode acquisition decreases examination time by approximately $25 \%$ in bone and lung SPECT studies compared to step-and-shoot mode $(\sim 2$ min per single-bed SPECT study), without compromising image quality and signal quantification. This SPECT sensitivity improvement also offers the perspective of more comfortable exams, with less motion artifacts, especially in painful or dyspneic patients 
Acknowledgements

Not applicable

\section{Authors' contributions}

VP, SR, and MS contributed to the conception and design of the study. VP, NM, FB, SR, MP, and MS contributed to the data acquisition and analysis. NM, SR, and MS contributed to the data interpretation. VP, NM, SR, MP, and MS contributed to the drafting and/or critical revision for important intellectual content. All authors read and approved the final manuscript

\section{Funding}

No funding

\section{Availability of data and materials}

The datasets used and/or analyzed during the current study are available from the corresponding author on reasonable request.

\section{Ethics approval and consent to participate}

All procedures performed in this study were in accordance with the ethical standards of the institutional research committee (CLEA). Formal ethics committee approval was not required for this retrospective analysis of the routine use of the SPECT/CT technology.

\section{Consent for publication}

Not applicable

\section{Competing interests}

The authors declare that they have no competing interests.

\section{Author details}

${ }^{1}$ GE Healthcare, 78530, 283 Rue de la Miniere, Buc, France. ${ }^{2}$ Department of Nuclear Medicine, Avicenne Hospital, HUPSSD, APHP, Paris, France. ${ }^{3}$ Inserm, Institut Curie, Laboratoire d'Imagerie Translationnelle en Oncologie, Orsay, France.

Received: 28 February 2020 Accepted: 5 January 2021

Published online: 02 February 2021

\section{References}

1. Ljungberg M, Pretorius PH. SPECT/CT: an update on technological developments and clinical applications. Br J Radiol. 2018;91:20160402.

2. Kim J, Lee H-H, Kang Y, Kim TK, Lee SW, So Y, et al. Maximum standardised uptake value of quantitative bone SPECT/CT in patients with medial compartment osteoarthritis of the knee. Clin Radiol. 2017;72:580-9.

3. Beck M, Sanders JC, Ritt P, Reinfelder J, Kuwert T. Longitudinal analysis of bone metabolism using SPECT/CT and 99mTCdiphosphono-propanedicarboxylic acid: comparison of visual and quantitative analysis. EJNMMI Res [Internet]. 2016 [cited 2016 Oct 10];6. Available from: http://www.ncbi.nlm.nih.gov/pmc/articles/PMC4963336/

4. Ljungberg M. Absolute quantitation of SPECT studies. Semin Nucl Med. 2018;48:348-58.

5. Cao Z, Maunoury C, Chen CC, Holder LE. Comparison of continuous step-and-shoot versus step-and-shoot acquisition SPECT. J Nucl Med Off Publ Soc Nucl Med. 1996;37:2037-40.

6. Thibault F, Bailly M, Le Rouzic G, Metrard G. Clinical evaluation of General Electric new Swiftscan solution in bone scintigraphy on Nal-camera: a head to head comparison with Siemens Symbia. PLoS One. 2019;14:e0222490.

7. White D, Lawson RS. A Poisson resampling method for simulating reduced counts in nuclear medicine images. Phys Med Biol. 2015:60:N167-76.

8. Dickinson L, Ahmed HU, Allen C, Barentsz JO, Carey B, Futterer JJ, et al. Scoring systems used for the interpretation and reporting of multiparametric MRI for prostate cancer detection, localization, and characterization: could standardization lead to improved utilization of imaging within the diagnostic pathway? J Magn Reson Imaging JMRI. 2013;37:48-58.

9. Landis JR, Koch GG. An application of hierarchical kappa-type statistics in the assessment of majority agreement among multiple observers. Biometrics. 1977;33:363-74.

10. Altman DG, Bland JM. Measurement in medicine: the analysis of method comparison studies. J R Stat Soc Ser Stat. John Wiley \& Sons, Ltd; 1983:32:307-17.

11. Munn Z, Jordan Z. The effectiveness of interventions to reduce anxiety, claustrophobia, sedation and non-completion rates of patients undergoing high technology medical imaging. JBI Libr Syst Rev. 2012;10:1122-85.

12. Buechel RR, Herzog BA, Husmann L, Burger IA, Pazhenkottil AP, Treyer V, et al. Ultrafast nuclear myocardial perfusion imaging on a new gamma camera with semiconductor detector technique: first clinical validation. Eur I Nucl Med Mol Imaging. 2010;37:773-8.

13. Bailly M, Le Rouzic G, Metrard G, Ribeiro MJ. Faster acquisition for dopamine transporter imaging using swiftscan step and shoot continuous SPECT without impairing visual and semiquantitative analysis. Front Med. 2020;7:235.

14. Matsutomo N, Takano E, Yamamoto T, Sato E. Continuous repetitive data acquisition with (123)-FP-CIT SPECT: effects of rotation speed and rotation time. J Nucl Med Technol. 2019;47:319-25.

15. Dickson J, Ross J, Vöö S. Quantitative SPECT: the time is now. EJNMMI Phys. 2019;6:4.

\section{Publisher's Note}

Springer Nature remains neutral with regard to jurisdictional claims in published maps and institutional affiliations. 